\title{
THE ROLE OF COBALT HYDROXIDE IN DEACTIVATION OF THIN FILM CO-BASED CATALYSTS FOR SODIUM BOROHYDRIDE HYDROLYSIS
}

M. Paladini, G.M. Arzac*, V. Godinho, D. Hufschmidt, M.C. Jiménez de Haro, A.M. Beltrán $^{+}$, A. Fernández ${ }^{*}$

Instituto de Ciencia de Materiales de Sevilla (CSIC-Univ. Sevilla), Avda. Américo Vespucio 49, 41092-Sevilla, Spain.

${ }^{+}$Current adress: Departamento de Ingeniería y Ciencia de los Materiales y del Transporte. Escuela Técnica Superior de Ingeniería, Camino de los Descubrimientos s/n, 41092 Sevilla, Spain.

* Corresponding authors: asuncion@icmse.csic.es (A. Fernández), gisela@icmse.csic.es (G.M Arzac)

\begin{abstract}
Deactivation of a Co catalyst prepared as thin film by magnetron sputtering was studied for the sodium borohydride (SB) hydrolysis reaction under different conditions. Under high SB concentration in single run experiments, the formation of a B-O passivating layer was observed after 1.5 and $24 \mathrm{~h}$ use. This layer was not responsible for the catalyst deactivation. Instead, a peeling-off mechanism produced the loss of cobalt. This peeling-off mechanism was further studied in cycling experiments (14 cycles) under low SB concentrations. Ex-situ study of catalyst surface after use and solid reaction products (precipitates) was performed by X-Ray photoelectron spectroscopy (XPS), transmission electron microscopy (TEM) and scanning transmission electron microscopy (STEM). The presence of cobalt hydroxide and oxyhydroxide was detected as major components on the catalyst surface after use and as precipitates in the supernatant solutions after washing. Cobalt borate, cobalt carbonate and oxycarbonate
\end{abstract}


were also formed but in lesser amounts. These oxidized cobalt species were formed and further detached from the catalyst at the end of the reaction and/or during catalyst washing by decomposition of the unstable in-situ formed cobalt boride. Leaching of cobalt soluble species was negligible. Thin film mechanical detachment was also found but in a smaller extent. To study the influence of catalyst composition on deactivation processes, cycling experiments were performed with $\mathrm{Co}-\mathrm{B}$ and $\mathrm{Co}-\mathrm{C}$ catalysts, also prepared as thin films. We found that the deactivation mechanism proposed by us for the pure Co catalyst also occurred for a different pure Co (prepared at higher pressure) and the Co-B and Co-C samples in our experimental conditions.

\section{Keywords}

Hydrogen, sodium borohydride, Co thin film catalyst, deactivation, cobalt hydroxide/oxyhydroxide

\section{Introduction}

The use of hydrogen as energy carrier is recognized as one of the most promising solutions to replace the shrinking fossil fuel resources. However, for the development of the "hydrogen economy", several challenges related to production (as green as possible), transport, storage and combustion should be faced first. In this context, sodium borohydride $\left(\mathrm{NaBH}_{4}, \mathrm{SB}\right)$ has gained a lot of attention as hydrogen storage material because it combines lightweight with high hydrogen content (10.8 wt. \%) [1-6]. Stabilized aqueous sodium borohydride solutions can be considered liquid-fuels, which can release hydrogen through reaction (1) at appreciable rates in the presence of suitable catalysts.

$$
\mathrm{BH}_{4}{ }^{-}(a q)+4 \mathrm{H}_{2} \mathrm{O}(l) \rightarrow 4 \mathrm{H}_{2}(g)+\mathrm{B}(\mathrm{OH})_{4}{ }^{-}(a q)
$$


Among catalysts noble metals are very efficient for the catalytic hydrolysis of SB but their scarcity and high cost leads to the search of alternatives. In this direction, cobalt based materials have been intensively investigated because of their relative low cost and high activity [4-6]. According to literature there are many papers reporting the preparation of Co based catalysts with varying activity [4-10]. However, the major drawback of cobalt is that it deactivates upon use [5, 11-12]. Thinking of practical applications, the catalyst durability is an important requirement to be fulfilled. The improvement of cobalt stability and durability is considered one of the most challenging issues in the field of sodium borohydride hydrolysis. The main difficulty in the study of reaction (1) is that SB is a very strong reducing agent $(-1.24 \mathrm{~V}$ vs $\mathrm{SHE}$ at $\mathrm{pH} 14)$ and also a boron donor, which strongly changes the catalysts surface under operation [4, 11]. These changes cannot be directly evidenced by ex-situ characterization, because this in-situ formed surface is unstable and is again modified during reaction quenching (extraction, washing and drying of catalyst) [4]. Despite this difficulty different deactivation mechanisms have been proposed so far. The most accepted mechanism is the formation of a thick passivation layer of B-O based compounds (reaction byproducts) on catalysts surface [11-13]. In principle, this layer cannot be avoided and was proposed as part of the active phase $\left(\mathrm{Co} @ \mathrm{~B}_{\alpha} \mathrm{O}_{\beta}(\mathrm{OH})_{\gamma}\right)$ by other authors [5]. After reaction, extraction and washing processes contribute to change the surface stoichiometry leading to a less active catalyst $[5,11]$. In view of this, ex-situ washing with diluted acid was proposed as an efficient method to eliminate this B-O layer (basic in nature) and by this recover the catalyst activity [11-12]. Low temperature calcination was also reported as an efficient method to eliminate water from this layer to recover the catalysts original activity [14]. Alternatively, cobalt can be combined with other metals in order to reduce its sensitivity to borates adsorption. In this direction, $\mathrm{Cu}-\mathrm{Co}$ 
based catalysts have demonstrated to be stable against B-O adsorption, avoiding the formation of the passivation layer and thus being more resistant [8].

Apart from the formation of a borate layer, other proposed deactivation mechanisms are catalyst aggregation and leaching of cobalt active phase. However the effect of the corrosive nature of the (NaOH-stabilized) SB solutions is not usually addressed.

In this paper we study the deactivation processes occurring in a Co based catalyst prepared as thin film by magnetron sputtering (MS) upon SB hydrolysis. We have selected the MS method to prepare a supported pure Co catalyst without the presence of any boron at medium pressure on flexible polytetrafluoroethylene (PTFE) membranes, on Ni foam and Si according to our previous reports [15-16]. The use of MS as a tool to prepare supported catalytic coatings also improves the separation of the catalyst from the reaction solutions for further analysis. The PTFE membranes facilitate surface characterization and routine in-lab cobalt quantification without the interference of $\mathrm{Ni}$ (see experimental section below). Durability was studied in two different conditions: high SB concentration conditions in single runs of 1.5 and $24 \mathrm{~h}$ and low SB concentration conditions in cycling (start-stop) experiments. The catalyst was characterized before and after use together with the supernatant solutions. The role of the B-O passivating layer, leaching of soluble Co species and the formation of oxidized cobalt species in connection to deactivation is herein discussed based on our results and those found in literature.

\section{Experimental Section}

\subsection{Catalyst preparation}

The Co catalyst in the form of a thin film was prepared by MS on the basis of our previous work [15-16]. The coating was deposited using a 2 inch diameter magnetron 
from AJA in magnetic target configuration, from a pure cobalt target (Kurt J. Lesker 99.95\% pure, $1 \mathrm{~mm}$ thickness) under pure argon atmosphere. The power applied to the Co target was $50 \mathrm{~W}$ in DC mode $\left(\mathrm{Co}_{50}\right)$. The base pressure before deposition was $10^{-6}$ mbar and the working pressure was $2.8 \times 10^{-2}$ mbar. The catalyst was deposited as a thin film directly on a commercial porous PTFE-membrane (expanded PTFE on a polypropylene support, Pall Life Sciences 8"x10") and on Ni foam (Goodfellow $1.6 \mathrm{~mm}$ thick, $95 \%$ porosity, 20 pores per $\mathrm{cm}$ ) for catalytic studies. For coating characterization substrates of Si (100) were employed. After deposition the PTFE membrane was cut into $1 \mathrm{~cm} \times 1 \mathrm{~cm}$ pieces and the amount of Co was quantified (see below). Ni foam was cut into ca. $0.5 \mathrm{~cm} \times 0.5 \mathrm{~cm}$ pieces before deposition and grouped. Each group was weighted before and after deposition to obtain the total mass of catalyst deposited. Before each synthesis the $\mathrm{Ni}$ foam pieces were washed in an ultrasonic bath successively with distilled water, ethanol/acetone (1:1), $\mathrm{HCl} 0.1 \mathrm{M}$ and again distilled water, ethanol and acetone to finally be dried in air for 24 hours. No previous treatment was done in PTFE membrane. The Si substrates were cleaned with acetone and dried in a nitrogen flow.

\subsection{Catalyst and reaction products characterization}

X-ray photoelectron spectroscopy (XPS) measurements were recorded with a SPECS radiation with $35 \mathrm{eV}$ pass energy at normal emission take off angle. The spectra were calibrated with the position of the $\mathrm{C}(1 \mathrm{~s})$ signal (from contamination) at $284.6 \mathrm{eV}$ validated with the characteristic position of oxidized Co $\left(2 \mathrm{p}_{3 / 2}\right)$ at $780.9 \mathrm{eV}$. Quantification was carried out with the CasaXPS ${ }^{\circledR}$ program provided by the SPECS Company.

For the quantification of cobalt in membrane deposited catalysts, the colorimetric nitroso-R salt (NRS) method was employed [17]. A piece of $1 \mathrm{~cm}^{2}$ of supported catalyst 
was dissolved in $1 \mathrm{~mL} 1 \mathrm{M} \mathrm{HCl}$. Then $1 \mathrm{~mL} 10 \mathrm{wt}$. \% sodium acetate, $9 \mathrm{~mL} 0,065 \mathrm{M}$ $\mathrm{NaOH}$ and $0.5 \mathrm{~mL} 0.011 \mathrm{M}$ NRS solutions were added. The resulting solution was shaken vigorously and then $1 \mathrm{~mL}$ was diluted to $5 \mathrm{~mL}$ and transferred to a quartz cuvette (SUPRASIL ${ }^{\circledR}, 10 \mathrm{~mm}$ path-length and $10 \mathrm{~mm}$ width). Absorbance at $500 \mathrm{~nm}$ was immediately measured in a double beam spectrophotometer (UV-VIS scanning spectrophotometer 2101PC, SHIMADZU). Co concentration was calculated by using the calibration curve (shown in Fig. S1, as supporting info) obtained with samples of known Co concentration. In those cases, in which absorbance exceeded the linear range, the piece of $1 \mathrm{~cm}^{2}$ of supported catalyst was dissolved in $3 \mathrm{mLof} 1 \mathrm{M} \mathrm{HCl}$. For quantification of very small amounts of Co, inductively coupled plasma atomic emission spectroscopy (ICP-AES) measurements were performed (at CITIUS, Univ. Seville).

Scanning electron microscopy (SEM) was performed with a high resolution FEG microscope HITACHI S4800 equipped with an energy dispersive X-ray spectroscopy (EDX) detector (Bruker X-Flash 4010). Coatings were analyzed directly on the Ni foam or PTFE membrane in top view observations. For cross section views cleaved samples from coatings grown on $\mathrm{Si}$ substrates were used. The thickness of the coatings was evaluated from the SEM cross-section measurements. SEM analyses were also done on catalysts surface after extraction from reaction media and on recovered precipitates from supernatant solutions after cycling experiments (see details in the results and discussion section below).

These recovered precipitates were also studied by scanning-transmission electron microscopy (STEM) techniques at the Laboratory of Nanoscopies and SpectroscopiesLANE at the ICMS using a FEI Tecnai F30 microscope operated at $300 \mathrm{keV}$. Samples were studied by conventional bright-field (BF), selected area electron diffraction 
(SAED), and high-resolution TEM (HRTEM) imaging. Simulation and indexing of diffraction patterns obtained from Fast Fourier Transforms (FFT) of high resolution images or directly from SAED were carried out with the software JEMS [http://www.jems-saas.ch/]. For high resolution compositional studies, electron energyloss spectroscopy spectrum-images (EELS-SIs) were recorded with a GATAN GIF Quantum energy filter coupled to the scanning TEM high-angle annular dark field (STEM-HAADF) mode. Data acquisition and processing were done with Digital Micrograph [http://www.gatan.com] software.

\subsection{Catalytic tests}

Catalyst deactivation was studied under high and low SB concentration conditions.

\subsubsection{High SB concentration conditions, single run experiments.}

To study deactivation under high SB concentration conditions, samples (catalysts supported on membranes, $1 \mathrm{~cm}^{2}$ ) were immersed in $1 \mathrm{~mL} 19$ wt.\% SB, 4.5 wt.\% $\mathrm{NaOH}$ solutions during 1.5 and $24 \mathrm{~h}$ (independent experiments) at room temperature (not thermostatized). After reaction catalysts were washed with deionized water and dried at room temperature. Activity was tested, for pristine and used samples, at $60{ }^{\circ} \mathrm{C}$ (in a thermostatic bath) by adding $1 \mathrm{~mL} 3.6 \mathrm{wt}$ \% $\mathrm{SB}$ (stabilized in $4.5 \mathrm{wt} \% \mathrm{NaOH}$ ) to the catalytic membranes cut into small pieces.

\subsubsection{Low SB concentration conditions, cycling (multi-run) experiments.}

Deactivation was also studied for $\mathrm{Ni}$ foam supported catalysts under low SB concentration conditions upon cycling (14 cycles). Each run consisted in adding of $1 \mathrm{~mL}$ 3.6 wt. $\% \mathrm{SB}$ (stabilized in 4.5 wt. $\% \mathrm{NaOH}$ ) to the supported catalyst. Runs were conducted at $25{ }^{\circ} \mathrm{C}$ (in a thermostatic bath). After each run catalyst was extracted and 
washed with deionized water, ethanol and dried at room temperature. Supernatant solutions were separated, accumulated and stored for further studies.

To quantify the amount of cobalt lost in each cycle a group of Ni foam supported $\mathrm{Co}_{50}$ catalyst was exposed to $1 \mathrm{~mL} 3.6$ wt.\% $\mathrm{SB}$ (stabilized in 4.5 wt.\% $\mathrm{NaOH}$ ). After reaction, the supernatant solution was extracted and separated (SS1). The catalyst was washed several times with pure water and the washing solutions were also separated (WS2). Both SS1 and WS2 solutions were mixed together and filtered. The amount of cobalt in the precipitates was measured by ICP-AES by previously dissolving in $5 \mathrm{~mL}$ $1 \mathrm{M} \mathrm{HCl}$. The amount of soluble cobalt in the supernatant was measured also by ICPAES. Form comparison, the same experiment was done by exposing a group of $\mathrm{Ni}$ foam supported $\mathrm{Co}_{50}$ catalyst to $1 \mathrm{~mL} 4.5$ wt. $\% \mathrm{NaOH}$ solution (a boron-free solution).

\section{Results and discussion}

\subsection{High SB concentration conditions in single runs.}

It is known that Co catalysts deactivate upon reaction with SB. It is generally accepted that the adsorption of borate by-products forms a passivating layer, which causes catalyst deactivation. To study the role of adsorbed surface borates in deactivation of thin film $\mathrm{Co}_{50}$ catalyst a first set of experiments was conducted under high SB concentration conditions (and thus high $\mathrm{B}(\mathrm{OH})_{4}{ }^{-}$or $\mathrm{BO}_{2}{ }^{-}$concentration conditions). The catalyst was exposed to SB during single runs of variable duration (1.5 and 24h) and then the activity was tested (after ex-situ washing and drying). To facilitate surface characterization (XPS) and quantification of Co (photometric method) these experiments were carried out using the PTFE supported catalyst only.

XPS measurements were performed on catalyst exposed during 1.5 and $24 \mathrm{~h}$ to $19 \mathrm{wt} . \%$ SB solutions (NaOH-stabilized) in comparison to the fresh one. Normalized spectra are 
shown in Fig. 1. Before and after exposure to SB samples show the presence of oxidized cobalt at $780.9 \mathrm{eV}$ and $786.2 \mathrm{eV}$ (satellite peak) in Co $2 \mathrm{p}_{3 / 2}$ level. The study of the B $1 \mathrm{~s}$ spectra of 1.5 and $24 \mathrm{~h}$ used samples shows the presence of the signal at $191.2 \mathrm{eV}$ attributed to $\mathrm{B}-\mathrm{O}$ species (mainly in the form of $\mathrm{BO}_{2}{ }^{-}$) not observed on the fresh sample $[4,13]$. This is consistent with the idea that borates adsorb strongly on Co surface upon use. The study of the $\mathrm{Na}(1 \mathrm{~s})$ level shows the signal at $1071.5 \mathrm{eV}$ after $24 \mathrm{~h}$ use. It seems that sodium incorporates in the B-O network at high exposure times, probably to balance negative charges of borate anions. Other authors found the incorporation of sodium on a Ni based catalyst for the same reaction [18].

Quantification of the XPS spectra (see Fig. 2 and Table S1) shows that surface amount of Co decreases upon exposure to SB with a concomitant increase in the amount of surface B. After the first increase in the amount of B at $1.5 \mathrm{~h}$, longer exposure to reach $24 \mathrm{~h}$ produces a further increase in the amount of surface borates ( 7.5 vs 10.6 at. \% of B) but not as high as the one occurred during the first $1.5 \mathrm{~h}$ period. Similar behavior occurs with cobalt, which reduces its surface amount from 20.5 to 13.6 at. $\%$ in the $1.5-24 \mathrm{~h}$ period. In this case, the decrease in the amount of surface cobalt after $24 \mathrm{~h}$ can also be explained by the increase in the amount of $\mathrm{Na}$, probably to balance the negative charge of surface borates. These results support the idea that the catalyst surface changes strongly after its first exposure to SB, but then, keeping it longer in solution for continuous use, does not further change surface composition significantly, according also to previous observations [5].

The activity of fresh $\mathrm{Co}_{50}$ catalyst was compared with those previously exposed during 1.5 and $24 \mathrm{~h}$ to 19 wt. \% SB solutions stabilized in 4.5 wt. \% $\mathrm{NaOH}$. Hydrogen generation rates (in $\mathrm{mL} \cdot \mathrm{min}^{-1}$ ) and mass of cobalt (photometric method) were measured in duplicate experiments and the results are shown in Table 1 . The activity of the fresh 
catalyst is moderate: $7.9 \mathrm{~L} \cdot \mathrm{min}_{\mathrm{H} 2}{ }^{-1} \cdot \mathrm{g}_{\mathrm{Co}}{ }^{-1}$. Higher activities were achieved in our previous work by varying deposition conditions (applied power, pressure, etc) and composition (Co-B and Co-C series) [16]. After $1.5 \mathrm{~h}$ of use the reaction rate decreases to reach $93 \%$ of the initial. The same decrease was found after $24 \mathrm{~h}$ use. Apparently, the adsorption of borates could not explain this exposure time independent decrease in activity: samples after 1.5 and $24 \mathrm{~h}$ show increasing amounts of boron in the form of borates and decreasing amounts of surface cobalt (20.5 vs. 13.6 at. \%). If the decrease in the amount of surface cobalt would be responsible for the loss in activity, the samples after $24 \mathrm{~h}$ exposure should be less active than after $1.5 \mathrm{~h}$. However, quantification of the remaining cobalt shows that after 1.5 and $24 \mathrm{~h}$ use the amount of active phase is around $89 \%$ of the initial. Under these conditions, it seems that the decrease of the reaction rates can be correlated to a decrease in the mass of cobalt within the experimental error. This is confirmed by the study of the activity per mass of $\mathrm{Co}\left(\mathrm{in}_{\mathrm{H} 2} \cdot \mathrm{min}^{-1} \cdot \mathrm{g}_{\mathrm{Co}}{ }^{-1}\right.$ ), which shows small variations (from 7.9 to 8.2 ) between the fresh catalyst and those exposed to $\mathrm{SB}$, indicating little or no dependence of the surface composition.

The catalyst was studied ex-situ by SEM/EDX after 1.5 and $24 \mathrm{~h}$ exposure to SB in comparison to the $\mathrm{Co}_{50}$ fresh sample (see Fig. 3). The fresh thin film catalyst covers efficiently the membrane support obtaining a conformal coating [15-16]. After $1.5 \mathrm{~h}$ of use the catalyst shows the formation of a layer with higher oxygen content than the fresh sample according to EDX. This layer spreads over the whole catalyst surface. Surprisingly, within $24 \mathrm{~h}$ operation the catalyst seems to recover its initial morphology and relative composition. According to XPS results (Fig. 2), the amount of boron in the form of borate increases with exposure time. If the layer found at $1.5 \mathrm{~h}$ use was exclusively attributed to B-O compounds, samples after $24 \mathrm{~h}$ would be more covered than those after $1.5 \mathrm{~h}$. This means that the observation of catalysts in top view by SEM 
does not permit to accurately account for the presence of this B-O layer. To explain the recovery of the initial morphology after $24 \mathrm{~h}$ use, we propose a peeling-off effect, which occurs during catalyst operation or during washing (both effects indistinguishable with ex-situ characterization). This peeling- off effect eliminates surface layers and exposes a renewed catalyst surface after $24 \mathrm{~h}$ use. This effect will be discussed more in detail in the following sub-section (cycling experiments).

\subsection{Low SB concentration conditions in cycles.}

In the previous section the role of the formation of a B-O layer on catalyst surface was studied in relation to the catalyst deactivation. We found that this passivating layer was not responsible of catalyst deactivation in our conditions. Instead of this, it was attributed to a loss of cobalt. SEM images showed a sort of peeling-off mechanism responsible of loss of active phase.

Cycling experiments are ideal for the study of loss of active phase, because the active phase (together with the reaction products) is removed between cycles. For this reason, 14 start-stop cycles were performed on the Co catalyst supported on Ni foam under low concentration SB solutions (3.6 wt. \%) at $25{ }^{\circ} \mathrm{C}$. Under these conditions the concentration of $\mathrm{BO}_{2}{ }^{-}$at the end of the reaction is lower than with high $\mathrm{SB}$ concentrations (section 3.1). After each cycle resulting supernatant solutions were recovered and studied. Fig. 4 shows the kinetic results. The $\mathrm{Co}_{50}$ catalyst shows a clear decrease in reaction rates in respect to the initial upon cycling. After 14 cycles the reaction rate has reduced to around $14 \%$ of the initial one. To investigate the reason of deactivation and with the idea of eliminating the B-O basic layer, the catalyst was washed with diluted $\mathrm{HCl}\left(10^{-4} \mathrm{M}\right)$ after the $14^{\text {th }}$ cycle and activity again was tested $[5$, 11-12]. The activity of the acid washed catalyst is shown in Fig. 4 (open squares). No 
significant activity recovery was found, which indicates again that the formation of a BO passivating layer is not responsible of deactivation in our conditions.

The used $\mathrm{Co}_{50}$ catalyst was studied by SEM/EDX after 14 cycles, which results are shown in Fig. 5. Comparison with the fresh sample (inset, left), indicates that surface is covered by a layer, which also shows lamellar shaped hexagonal precipitates. This layer (indicated by arrows) has similar aspect of that usually attributed to B-O compounds [7$12,15]$. However, the formation of hexagonal, lamella-like precipitates was not reported before for this system. The shape and aspect of these precipitates resembles those reported before for oxidized cobalt species [19-20]. These compounds could be formed by the interaction of the highly corrosive reaction medium $\left(\mathrm{BO}_{2}^{-}+\mathrm{NaOH}\right)$ with the supported catalyst. EDX in Fig. 5 supports this idea, because the used catalyst contains oxygen in higher amounts than the fresh one. The presence of boron cannot be disregarded according to our previous XPS measurement. However, this element is quite difficult to be detected by EDX under our experimental conditions.

For a better comprehension of the interaction between the reaction medium with our $\mathrm{Co}_{50}$ thin film catalyst, we exposed fresh samples to the following liquid media: i) pure MilliQ ${ }^{\circledR}$ water, ii) 4.5 wt. $\% \mathrm{NaOH}$, iii) 4.5 wt. $\% \mathrm{NaOH}+\mathrm{NaBO}_{2}$ and iv) 3.6 wt.\% $\mathrm{SB}+$ 4.5 wt.\% $\mathrm{NaOH}$ (reaction solution). Solution iii) was obtained by total conversion of solution iv) with a Co catalyst and simulates the reaction products. Fresh catalyst was exposed to the solutions during $25 \mathrm{~min}$ at $25^{\circ} \mathrm{C}$. For solutions i)-iii), the $\mathrm{H}_{2}$ bubbling was simulated by immersing into an ultrasonic bath. Fig. 6 shows SEM images of the $\mathrm{Co}_{50}$ sample after exposure to the solutions in comparison to the fresh one. It is clear that the catalyst surface is strongly modified upon contact with aqueous media, because in these conditions cobalt is unstable, and oxidizes to $\mathrm{Co}^{\mathrm{II}}$ (which can further react with $\mathrm{OH}^{-}$) according to Pourbaix diagram [21]. The catalyst after exposure to pure water has 
similar aspect to that usually attributed to the formation of the B-O layer $[7-12,15]$. Catalyst after reaction with solution iv) looks like a mixture of the sample exposed to i) pure water and ii) $\mathrm{NaOH}$ (a boron-free solution). This experiment shows again that solely SEM images in top view are not indicative of B-O compounds and that aqueous media strongly modify Co catalysts whether SB hydrolysis occurs or not. Fig. 6 also shows a higher magnification cross sectional SEM image of the Co catalyst after exposure to the reaction solution (iv). It clearly shows the fresh thin film with the precipitates on surface. EDX on the top view of precipitates again shows a high contribution of $\mathrm{O}$, indicating the possible formation of oxidized cobalt species. In principle, the formation of oxidized cobalt species should not be detrimental to the activity. It is known that cobalt oxides are active catalysts for SB hydrolysis via in-situ formation of $\mathrm{Co}_{\mathrm{x}} \mathrm{B}$ phases [22-24]. However, detachment of these loosely bound crystals would lead to the loss of mass of cobalt.

To study catalyst detachment, the supernatants (SS1) obtained after exposing the $\mathrm{Co}_{50}$ catalyst to solutions (i-iv) were analyzed. After each experiment catalyst was extracted and washed with milliQ ${ }^{\circledR}$ water. We found that during washing catalyst detachment occurred. For this reason washing solutions (WS2) were also kept for study. SS1 and WS2 solutions were mixed together and filtered using a membrane. Solid products were found in all cases. Photographs of membranes after filtration are also shown as supporting information (Fig. S2). Ochre precipitates and small dark particles can be distinguished visually on the filtering membranes. The characterization of the solid products by SEM (Fig. S2) indicates that the ochre precipitates consist mostly of hexagonal lamella shaped precipitates. Dark particles consist of fresh Co thin film detached from the support. Solid products generated by SB reaction solution (iv) are mostly hexagonal precipitates with little contribution of thin film detachment. High 
amounts of fresh thin film were found in solids obtained from solutions ii) and iii) probably detached by synergism of ultrasound and the highly corrosive medium. These experiments indicate that the thin film is mechanically not robust enough for this reaction and thus robustness should be improved. Plasma ICP measurements were done to quantify cobalt in supernatant solutions $(\mathrm{SS} 1+\mathrm{WS} 2)$ obtained from exposure of $\mathrm{Co}_{50}$ to solutions ii) and iv) after filtration (pure leaching effect). The solid products recovered after filtration were dissolved and the amount of cobalt was also quantified (detachment/precipitates effect). Results are shown in Table S2, as supporting information. It was found that the amount of leached cobalt is negligible (less than $0.01 \%$ of the initial). However, the amount of solid cobalt was 2.8 wt. $\%$ of the initial after exposure to solution ii) and $5.3 \mathrm{wt} . \%$ after exposure to reaction solution iv). This result confirms that losses of Co-based solid products occur upon exposure to single $\mathrm{NaOH}$ solutions as well as to $\mathrm{SB}+\mathrm{NaOH}$. However, exposure to $\mathrm{SB}$ solution produces higher (1.9 times) loss of cobalt.

Fig.7 shows a SEM image of the precipitates recovered after the 14 cycles thoroughly washed with milliQ ${ }^{\circledR}$ water to eliminate rests of the reaction solutions. Precipitates are similar in shape and structure to those observed on used catalysts surfaces and after exposure to solutions i-iv) (Figs. 5-6). EDX shows again high contribution of Co and O. The inset in Fig. 7 shows a macroscopic image of accumulated precipitates in aged supernatant solution after 14 cycles.

Recovered precipitates after the 14 cycles and washing with milliQ ${ }^{\circledR}$ water were dispersed in ethanol and dropped onto carbon film TEM grids for further analysis. EELS is a powerful analytical tool for the study and quantification of light elements (especially B) [13]. Fig. 8a shows a STEM-HAADF image of the precipitates presenting, at higher magnification, acicular and lamella shaped microstructures similar 
to SEM images above. The recording of STEM-EELS spectrum-imaging data in the marked zone (Fig. 8b) allows the extraction of the $\mathrm{Co}-\mathrm{L}_{2,3}, \mathrm{O}-\mathrm{K}$ and $\mathrm{C}-\mathrm{K}$ majority signals and their quantification. Oxygen, cobalt and carbon elemental maps are shown in Fig. 8 c, d and e, respectively. The RGB color map is also shown in Fig. 8f. No boron signal was detected. Compositional maps show that precipitates contain mostly Co and O. This indicates again the presence of oxidized cobalt species. To gain more information about the composition and nature of the precipitates, the structural characterization by TEM is presented in Fig. 9. Conventional Bright field image (Fig. 9a) shows an overview of the samples with the characteristic acicular flakes, which appeared crystalline at higher magnification, as the HRTEM image shows (Fig. 9b). SAED pattern acquired at the central area of the flake is shown inset of Fig. $9 \mathrm{~b}$ and reveals a polycrystalline structure. More information about the crystalline structure can be obtained by the analysis of small and thin regions of the nanoflakes by the digital diffractogram obtained by FFT, as it has been done for the square area marked in b). The corresponding FFT is shown in Fig. 9c. Distances and angles between spots have been measured and indexed according to the simulated electron diffraction pattern (Fig. 9d) obtained by taking reference data from the X-Ray Diffraction (XRD) file ICCD 00$030-0443$ of the hexagonal $\beta-\mathrm{Co}(\mathrm{OH})_{2}$ crystalline phase. The simulation indicates the presence of this hexagonal cobalt hydroxide phase under the [110] zone axis confirming the presence of cobalt hydroxide in the recovered precipitates. Fig. S3 in supporting information also shows a comparison between experimental and simulated (for hexagonal $\mathrm{Co}(\mathrm{OH})_{2}$ phase) patterns of the SAED. Plasma ICP measurements were done to detect $\mathrm{B}$ in precipitates, but the amounts obtained are not significantly higher than in blank solutions to be considered reliable ( $\mathrm{B}$ is very difficult to detect in liquid media because borosilicate glassware contains significant amounts of boron that will leach) 
[25-26]. XPS measurements were also performed on precipitates and results are shown in Fig. S4. The study of the B 1s level show a very small and noisy signal at $193.1 \mathrm{eV}$ which can be attributed to the presence of borates. To discard the presence of contamination with rests of the reaction solution, XPS was measured on precipitates obtained upon exposure of Co catalyst to the $\mathrm{NaBO}_{2}+\mathrm{NaOH}$ solution (solution iii)). No boron was detected in this case. These results demonstrate that the presence of boron in precipitates after reaction cannot be disregarded, but occurs in small scale.

To clarify the composition of reaction precipitates on catalyst surface and after detachment, XRD and Raman spectroscopy measurements were performed and the results were compared with references. PTFE membrane supported $\mathrm{Co}_{50}$ catalyst was characterized after a single cycle (using $1 \mathrm{~mL} 3.6$ wt. \% SB solution at $25{ }^{\circ} \mathrm{C}$ ). Recovered precipitates were also analyzed and results are shown as supporting information. Fig. S5. shows XRD measurements performed on used catalyst and on recovered precipitates. Used catalyst was detached from the support mechanically by us after use and deposited on a Si plate (zero diffraction). Reaction precipitates were transferred to another Si plate after filtering. Both diffractograms (used catalyst and reaction precipitates) show similar information confirming that the identity of the precipitates on catalyst surface and in supernatant solution is the same. The diffraction peaks at $28.4^{\circ}$ are attributed to the $\mathrm{Si}$ plate. The broad peaks in the range $40-50^{\circ}$ are attributed to hcp and/ cubic metallic Co phases [15-16]. Precipitates show a strong reflection at $19^{\circ}$, which can be assigned to the $\beta-\mathrm{Co}(\mathrm{OH})_{2}$ phase (ICDD 00-030-0443) in accordance to TEM/SAED [27]. The diffraction peak at $25.3^{\circ}$ could indicate the presence of $\mathrm{Co}\left(\mathrm{BO}_{2}\right)_{2}$ (ICDD 01-077-0047) and/or $\mathrm{CoCO}_{3}$ (ICDD 01-078-0209). The presence of cobalt oxyhydroxide (CoOOH, ICDD 01-073-1213) cannot be disregarded. Raman spectra confirm these results. The fresh catalyst (Fig. S6) shows Raman bands at 
694,528 and $488 \mathrm{~cm}^{-1}$, which can be assigned to surface and intra-columnar $\mathrm{Co}_{3} \mathrm{O}_{4}$ and CoO (metallic Co cannot be seen by Raman spectroscopy) [28]. After use, the $\mathrm{Co}_{50}$ catalyst shows a broad signal in the range $635-483 \mathrm{~cm}^{-1}$, which can be either assigned to $\mathrm{Co}(\mathrm{OH})_{2}$ and $\mathrm{CoOOH}[27]$. The analysis of the Raman spectra of recovered precipitates confirms the presence of $\mathrm{Co}(\mathrm{OH})_{2}$ (the broad band in the $245-140 \mathrm{~cm}^{-1}$ range is indicative of $\left.\mathrm{Co}(\mathrm{OH})_{2}\right)$ and the peaks at 640 and $502 \mathrm{~cm}^{-1}$ show the presence of $\mathrm{CoOOH}$ [27]. Cobalt oxyhydroxide must be formed by further oxidation of the $\mathrm{Co}(\mathrm{OH})_{2}$ in solution under the presence of oxygen as reported in literature [29]. The presence of $\mathrm{Co}\left(\mathrm{BO}_{2}\right)_{2}$ and $\mathrm{Co}_{2} \mathrm{CO}_{3}(\mathrm{OH})_{2}$ cannot be disregarded [30-31].

Based on characterization, we conclude that precipitates are mostly composed of cobalt hydroxide and cobalt oxyhydroxide. Cobalt borate, cobalt carbonate and cobalt oxycarbonate are present but as minor components.

\subsection{Deactivation mechanism.}

Despite the experimental evidence of the formation of $\mathrm{B}-\mathrm{O}$ based compounds on catalyst surface, no correlation was found with catalyst deactivation in our conditions. According to our results the main deactivation mechanism for the thin film $\mathrm{Co}_{50}$ catalyst in single run as well as in cycling experiments is the loss of the cobalt. Cobalt hydroxide and oxyhydroxide (together with cobalt borate, cobalt carbonate and oxycarbonate as minor components) form on the catalyst surface after use and detach during catalyst washing, contributing to around 5 wt. \% of loss of active phase in each cycle. Leaching of soluble Co base species is negligible in our conditions contributing to less than 0.01 $\%$ of Co loss in one cycle. Fresh thin film (mechanical) detachment was also found responsible of loss of active phase, but in lesser extent. No evidence of catalyst aggregation was found in our conditions. 
The remaining question is: how do these oxidized cobalt species form? Based on the results discussed above, it becomes clear that the interaction of aqueous media with cobalt produces a cobalt-oxygen based layer because Co is intrinsically unstable. However, it was found that these cobalt hydroxide and oxyhydroxide were detached in higher amounts after reaction with SB than in alkaline $\mathrm{NaOH}$ solution. The key is that oxidized cobalt species are not stable under such a strong reducing agent as SB. [22-24]

Fig. 10 shows a scheme of the whole process. Before use (State I) Co catalyst is covered by a small oxide layer $\left(\mathrm{Co}_{3} \mathrm{O}_{4} / \mathrm{CoO}\right)$. During the first run (State II), SB reduces catalyst surface to form cobalt borides $\left(\mathrm{Co}_{\mathrm{x}} \mathrm{B}\right)$. At the end of the reaction, and/or after catalyst washing (State III), the $\mathrm{Co}_{\mathrm{x}} \mathrm{B}$ decomposes reacting with water and oxygen to form cobalt hydroxide, cobalt oxyhydroxide and borates. [22-25] Sodium borate is soluble in water and most borates dissolve into the washing solution. Part of the borates remains adsorbed on catalyst surface because cobalt borate is insoluble in water and forms part of a thin veil that covers the catalyst surface [13, 32]. Cobalt carbonate and oxycarbonate are also formed but in minor amounts. Those Co based solids are loosely bound to the catalyst surface and detach. The amount of cobalt detached in the form of precipitates after a single cycle is around $5 \mathrm{wt} . \%$ of the initial. After catalyst drying (State IV), those cobalt hydroxides and oxyhydroxides, which remained on the surface, may lose water molecules. This could influence in the activity in the following run, which effect was not studied in this work [14]. At the beginning of the second run, sodium borohydride reduces catalyst the surface again, forming the $\mathrm{Co}_{\mathrm{x}} \mathrm{B}$ active phase (State V). If the whole cycle (State I to IV) is repeated $\mathrm{n}$ times, the remaining catalyst reaches state $\mathrm{N}$, with a smaller film thickness and thus less amount of cobalt. Similar mechanism would apply for fresh thin film detached particles or direct leaching of the Co layer. From these, cobalt borides could also be formed under SB strong reduction 
conditions. And then, if all SB is consumed (or if reaction is quenched) the oxidized cobalt based compounds would be finally formed.

\subsection{Effect of composition. Co-B and Co-C thin film catalysts.}

We extended the present study to other thin film catalysts prepared by us before [16]. Durability was tested upon cycling on another pure Co thin film $\left(\mathrm{Co}_{50}-\mathrm{hp}\right.$, prepared at higher pressure that the $\mathrm{Co}_{50}$ catalyst studied above), a $\mathrm{Co}-\mathrm{B}\left(\mathrm{Co}_{50} \mathrm{~B}_{100}\right)$ and a $\mathrm{Co}-\mathrm{C}$ $\left(\mathrm{Co}_{50} \mathrm{C}_{100}\right)$ catalyst [15-16]. Details of preparation are found as supporting information (description in section e) and Table S3). Cycling experiments (Fig.S7) show similar deactivation effect as described above for $\mathrm{Co}_{50}$. Reaction rates decrease to reach $50 \%$ and $10 \%$ of the initial in $\mathrm{Co}_{50} \mathrm{C}_{100}$ and $\mathrm{Co}_{50} \mathrm{~B}_{100}$ samples respectively after all cycles. The $\mathrm{Co}_{50}$-hp sample completely lost its activity in the $10^{\text {th }}$ cycle. No recovery in activity was found after washing the three samples with diluted acid. The study of catalyst surface by SEM after 13-14 cycles also shows the formation of surface precipitates with similar morphology and composition to the previously described (Fig.S8). Characterization of supernatant reaction solutions was also performed. It was found the presence of hexagonal precipitates in all cases. These results permit to extend deactivation mechanism found for the pure $\mathrm{Co}_{50}$ catalyst to the $\mathrm{Co}-\mathrm{B}$ and $\mathrm{Co}-\mathrm{C}$ samples and the $\mathrm{Co}_{50}$-hp catalyst. The formation of cobalt based oxidized species and further detachment during catalyst washing seems to be intrinsic to cobalt in our films.

\section{Conclusions}

In this work we studied the deactivation mechanisms on a pure $\mathrm{Co}_{50}$ thin film catalyst prepared by MS for SB hydrolysis. The use of Magnetron Sputtering as a tool to prepare supported catalytic coatings facilitated both, the characterization of catalyst surface and the separation of supernatant solutions for further analysis. Deactivation was studied in 
single run as well as in cycling experiments. Under both conditions we found that the formation of B-O compounds on catalyst surface was not responsible of deactivation. Instead of this, we observed the loss of the active phase (cobalt). Oxidized cobalt species were formed and further detached from catalyst surface after each use. Those oxidized species consists mainly of cobalt hydroxide and oxyhydroxide. Cobalt borate, cobalt carbonate and oxycarbonate also form but in lesser amounts. Little direct detachment of the fresh thin film and negligible leaching of soluble cobalt species were observed.

The cobalt oxidized species form by decomposition of the in-situ formed $\mathrm{Co}_{\mathrm{x}} \mathrm{B}$ once $\mathrm{SB}$ is consumed and during catalyst washing. Similar results were obtained with other Co-B and $\mathrm{Co}-\mathrm{C}$ thin film catalysts extending the herein proposed deactivation mechanism further from pure Co. Our observations address the interactions between the highly corrosive nature of the reaction solutions $(\mathrm{SB}+\mathrm{NaOH})$ and the non-noble nature of cobalt in our thin films and gives additional information to propose the in-situ formation of $\mathrm{Co}_{\mathrm{x}} \mathrm{B}$ according to previous works.

\section{Acknowledgements}

Financial support is acknowledged from the Spanish MINECO (grants CTQ2012-32519 and CTQ2015-65918-R, EU co-financed by FEDER), the Junta de Andalucia (grant PE2012-TEP862, EU co-financed by FEDER) and the CSIC (PIE-201460E018). AMB thanks to Talent-Hub Program funded by the Junta de Andalucía and the European Commission under the Co-funding of the $7^{\text {th }}$ Framework Program in the People Program (Marie Curie Special Action). TEM measurements were performed at the Laboratory of Nanoscopies and Spectroscopies LANE at the ICMS. The authors thank 
I. Rosa, O. Montes, J.M. Martinez Blanes, M.A. Avilés and M.R. Garcia for their very valuable technical support.

\section{References}

[1] U. B Demirci, O. Akdim, J. Andrieux, J. Hannauer, R. Chamoun, P. Miele, Fuel Cells, 10 (3), (2010) 335-350.

[2] S.S. Muir, X. Yao, Int. J. Hydrogen Energy, 36 (10), (2011) 5983-5997.

[3] B.H. Liu, Z.P. Li, J. Power Sources, 187 (2), (2009) 527-534.

[4] U.B. Demirci, P. Miele, Phys. Chem. Chem. Phys. 12, (2010),14651-14665.

[5] U.B. Demirci, P. Miele, P. Phys. Chem. Chem. Phys. 16 (15), (2014),6872-6885.

[6] N. Patel, A. Miotello. Int. J. Hydrogen Energy, 40 (3), (2015), 1429-1464.

[7] L. Wang, Z. Li, X. Liu, P. Zhang, G. Xie. Int. J. Hydrogen Energy, 40 (25), (2015), 7965-7973.

[8] S. Eugénio, U.B. Demirci, T. M. Silva, M.J. Carmezim, M.F. Montemor. Int. J. Hydrogen Energy, 41 (2016), 8438-8448.

[9] Y. Wang, T. Li, S. Bai, K. Qi, Z. Cao, K. Zhang, D. Wang. Int. J. Hydrogen Energy, 41(1), (2016), 276-284.

[10] Y. Wang, Y. Shen, K. Qi, Z. Cao, K. Zhang, S. Wu. Renewable Energy, 89, (2016), 285-294. 
[11] O. Akdim, U.B. Demirci, P. Miele. Int. J. Hydrogen Energy, 36 (2011), 1366913675.

[12] G.M. Arzac., D. Hufschmidt , M.C. Jiménez de Haro, A. Fernández, B.Sarmiento , M.A. Jiménez., M.M. Jiménez , Int. J. Hydrogen Energy, 37, (2012), 14373-14381.

[13] G.M. Arzac, T.C. Rojas, A. Fernández, ChemCatChem, 3(8), (2011), 1305-1313.

[14] D.W. Zhuang, H.B. Dai, Y.J. Zhong, L.X. Sun, P. Wang, Int. J. Hydrogen Energy, 40(30), (2015), 9373-9381.

[15]M. Paladini, G.M. Arzac, V. Godinho, M.C. Jimenez de Haro, A. Fernández, Appl.Catal.B: Environ., 158-159, (2014), 400-409.

[16] ] M. Paladini, V. Godinho, G.M. Arzac, M.C. Jimenez de Haro, A.M. Beltrán, A Fernández , RSC Adv. 6, (2016) 108611-108620.

[17] G. Wunsch Talanta, 26(I), (1979), 177-179.

[18] J.H. Kim., K.T. Kim, Y.M. Kang, H.S. Kim, M.S. Song, Y.J. Lee, P.S. Lee, J. Y. Lee, J. Alloys and Compds, 379, (2004), 222-227.

[19] M. Qorbani, N. Naseri, A.Z. Moshfegh, ACS Applied Materials and Interfaces, 7(21), (2015). 11172-11179.

[20] N. Naseri, A. Esfandiar, M. Qorbani, A.Z. Moshfegh, ACS Sustainable Chemistry \& Engineering 4, (2016), 3151-3159.

[21] M. Varón, I. Ojea-Jimenez, J. Arbiol, L. Balcells, B. Martínez, V.F. Puntes, Nanoscale, 5(6), (2013). 2429-36. 
[22] V.I. Simagina, O.V. Komova, A.M. Ozerova, O.V. Netskina, G.V. Odegova,

D.G. Kellerman, O.A Bulavchenco, A.V. Ishchenko, Appl. Catal. A, 394(1-2), (2011) 86-92.

[23]P.Krishan, K. Hsueh, S. Yim, Appl. Catal. B. Environ, 77, (2007), 206-214

[24] D. Swierczynski, L. Laversenne, A. Garron, S. Bennici, C. Goutaudier, P. Miele, A. Auroux, B. Bonnetot, Int J. Hydrogen Energy 34 (2009). 938-951.

[25] A. Ledieu, F. Devreux, P. Barboux, L. Sicard, O. Spalla, Journal of Non Crystalline Solids 343 (2004) 3-12.

[26] E. Kmiecik, B. Tomaszewska, K.Wątor, M. Bodzek, Environ Sci Pollut Res, 23 (2016), 11658-11667.

[27] J. Yang, H. Liu, W.N. Martens, R.L. Frost, J. Phys. Chem. C 114, (2010) 111-119.

[28] Jongsomjit, B.; Panpranot, J.; Goodwin, J. G., Jr. J. Catal. 204 (1), 2001, 98-109.

[29] Y. Zhu, H. Li, Y. Koltypin, A. Gedanken, J. Mater. Chem., 12, 2002, 729-733.

[30] S. C. Neumaira, R. Kaindlb, H. Huppertza, ChemInform 65b(11), 2011, 1311-1317

[31] Yang, Jing, Cheng, Hongfei, \& Frost, Ray L. Molecular and Biomolecular Spectroscopy, 78(1), (2011) 420-428.

[32]A.M. Beltrán, M. Paladini, V. Godinho, G.M. Arzac, M.C. Jimenez de Haro, Conference: 16th European Microscopy Conference, At Lyon, Volume: II, page 822, DOI: 10.1002/9783527808465.EMC2016.5347. 


\section{Figure captions}

Figure 1: XPS spectra (normalized) on the $\mathrm{Co}_{50}$ catalyst upon exposure to $19 \mathrm{wt}$ \% SB solution ( $\mathrm{NaOH}$-stabilized) during 1.5 and $24 \mathrm{~h}$ in comparison to the fresh one.

Figure 2: Quantification of the XPS spectra on the $\mathrm{Co}_{50}$ catalyst upon exposure to 19 wt.\% SB solution (NaOH-stabilized) during 1.5 and $24 \mathrm{~h}$ in comparison to the fresh one $(0 \mathrm{~h})$.

Figure 3: SEM images on the $\mathrm{Co}_{50}$ catalyst upon exposure to 19 wt.\% SB solution during 1.5 and $24 \mathrm{~h}$ in comparison to the fresh one. The insets correspond to EDX spectra (the presence of $F$ is attributed to the PTFE substrate).

Figure 4: Cycling experiments (3.6 wt. \% SB ) on $\mathrm{Co}_{50}$ catalyst supported on $\mathrm{Ni}$ foam. Retained rates (Reaction ratex100/Initial rate) as a function of cycle number.

Figure 5: SEM and EDX analysis of the Ni-foam supported $\mathrm{Co}_{50}$ catalyst surface after 14 cycles (3.6 wt. \%SB).

Figure 6: SEM images of the $\mathrm{Co}_{50}$ catalyst surface after exposure to: i) pure water (MilliQ®), ii) $\mathrm{NaOH}$, iii) $\mathrm{NaOH}+\mathrm{BO}_{2}^{-}$and iv) $\mathrm{SB}+\mathrm{NaOH}$ (reaction) solutions in comparison to the fresh one.

Figure 7: SEM image and EDX analysis on the precipitates obtained from the supernatant solutions over 14 cycles on $\mathrm{Co}_{50}$ catalyst.

Figure 8: STEM-HAADF-EELS analysis on the precipitates obtained from the supernatant solutions over 14 cycles on $\mathrm{Co}_{50}$ catalyst. a) and b) STEM-HAADF images; 
(c -f) STEM-EELS elemental maps for the square in b) ( $c$ for oxygen, $d$ for cobalt, e for carbon and $\mathrm{f}$ the color map $\operatorname{Red}(\mathrm{Co}) \operatorname{Green}(\mathrm{O}) \operatorname{Blue}(\mathrm{C}))$. Common scale bar c-f: $10 \mathrm{~nm}$

Figure 9: TEM and electron diffraction analysis on the precipitates obtained from the supernatant solutions over 14 cycles on $\mathrm{Co}_{50}$ catalyst.(a) bright field image; (b) HRTEM image; inset SAED pattern (c) FFT of the square area in the HRTEM image in b); (d) Simulated electron diffraction pattern for the hexagonal cobalt hydroxide phase under the [110] zone axis.

Figure 10: Scheme of the proposed deactivation mechanism 


\section{Table legends}

Table 1: Single run durability experiments on membrane supported $\mathrm{Co}_{50}$ catalyst upon exposure to 19 wt. \% SB solutions during 1.5 and $24 \mathrm{~h}$. 
TABLE 1

\begin{tabular}{|c|c|c|c|c|c|c|}
\hline Catalyst & $\begin{array}{c}\text { Exp } \\
\text { Time } \\
\text { (h) } \\
\end{array}$ & $\begin{array}{l}\text { Reaction rates } \\
\left(\mathrm{mL} \cdot \mathrm{min}^{-1} \cdot \mathrm{cm}^{-2}\right)\end{array}$ & $\begin{array}{l}\text { Mass of Co } \\
\left(\mathrm{mg}_{\mathrm{Co}} \mathrm{cm}^{-2}\right)\end{array}$ & $\begin{array}{c}\text { Retained } \\
\text { rate } \\
(\%) \\
\end{array}$ & $\begin{array}{c}\text { Retained } \\
\text { Mass of Co } \\
(\%) \\
\end{array}$ & $\begin{array}{c}\text { Activity } \\
\left(\mathrm{L}_{\mathrm{H} 2} \min ^{-1} \mathrm{~g}_{\mathrm{Co}}{ }^{-1}\right)\end{array}$ \\
\hline \multirow[t]{3}{*}{$\mathrm{Co}_{50}$} & 0 & 1.5 & 0.19 & 100 & 100 & 7.9 \\
\hline & $1.5^{\mathrm{a})}$ & 1.4 & 0.17 & 93 & 89 & 8.2 \\
\hline & $24^{\text {a) }}$ & 1.4 & 0.17 & 93 & 89 & 8.2 \\
\hline
\end{tabular}

a) Exposed catalysts were tested after ex-situ washing and drying 
Figure 1

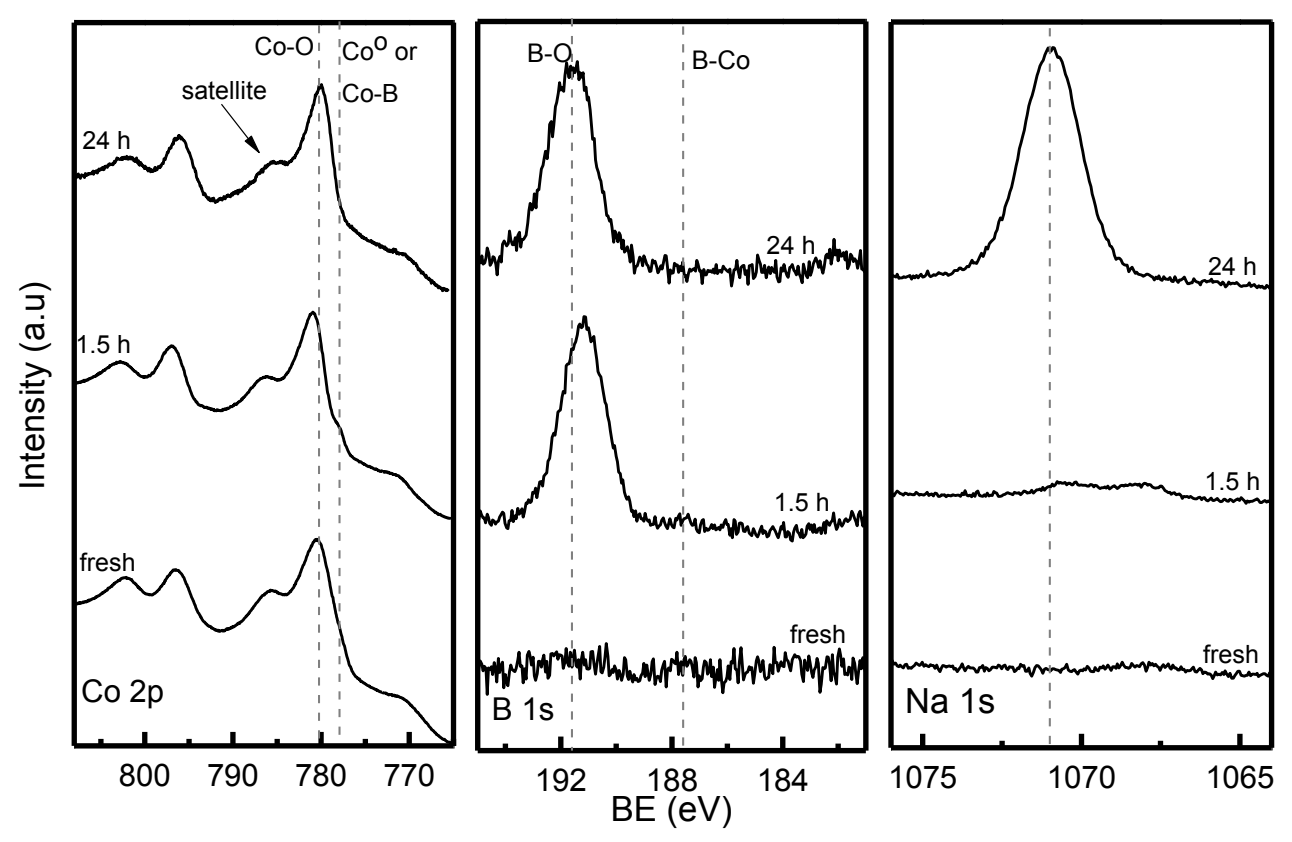


Figure 2

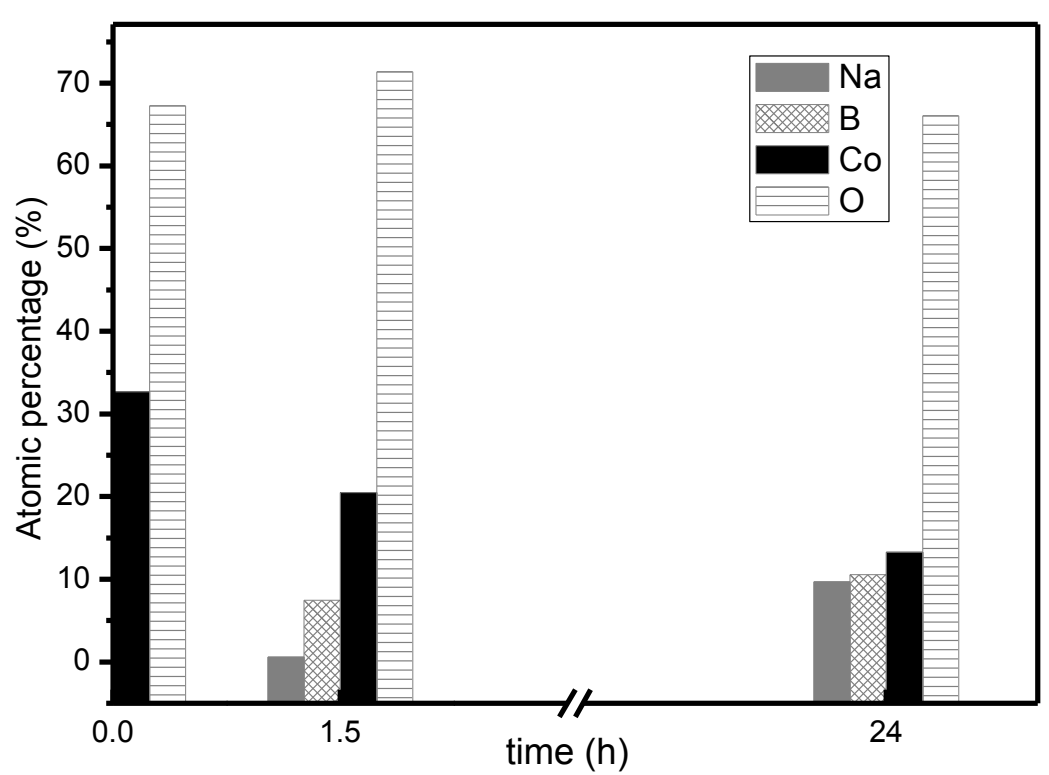




\section{Figure 3}
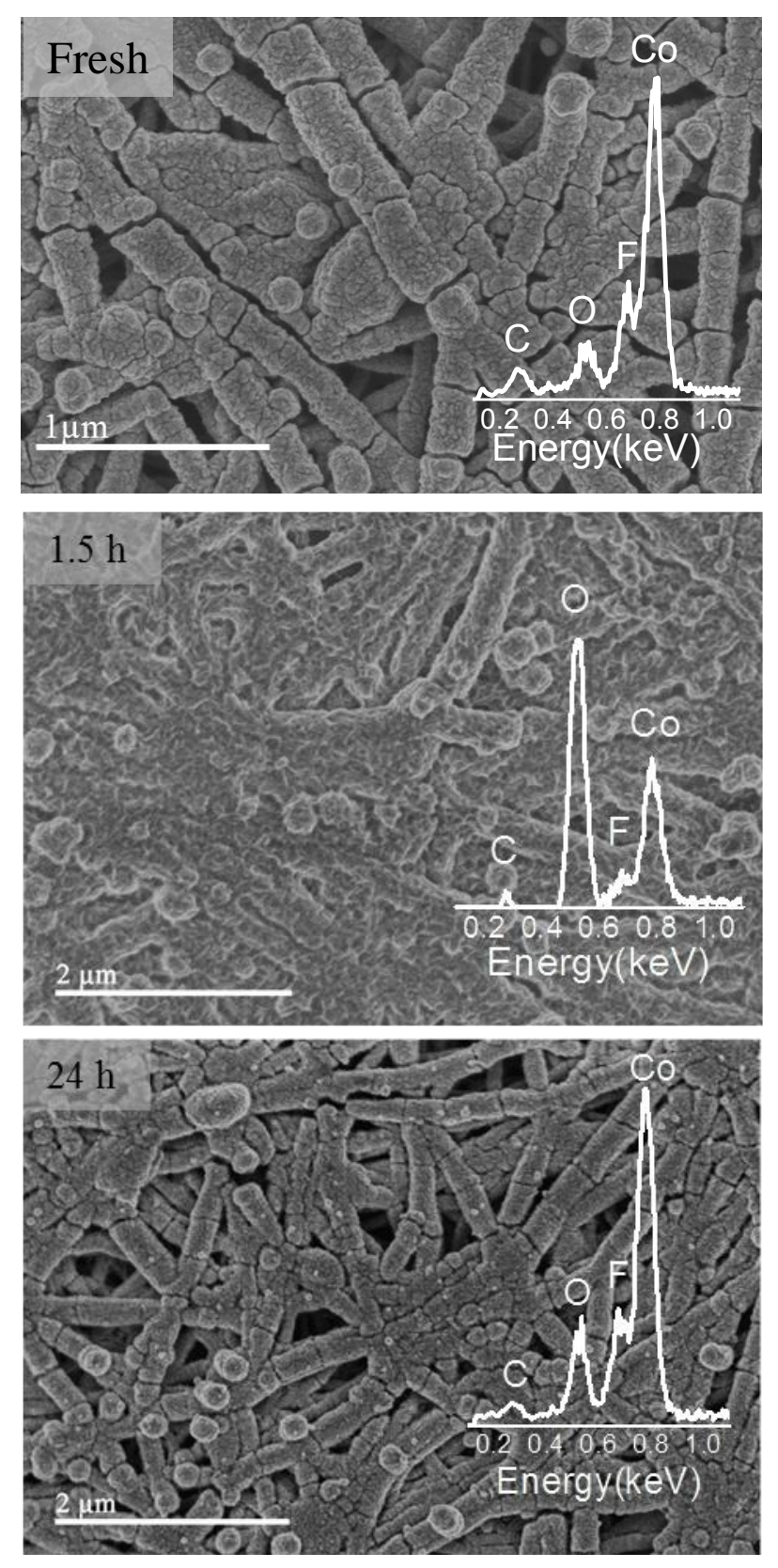
Figure 4

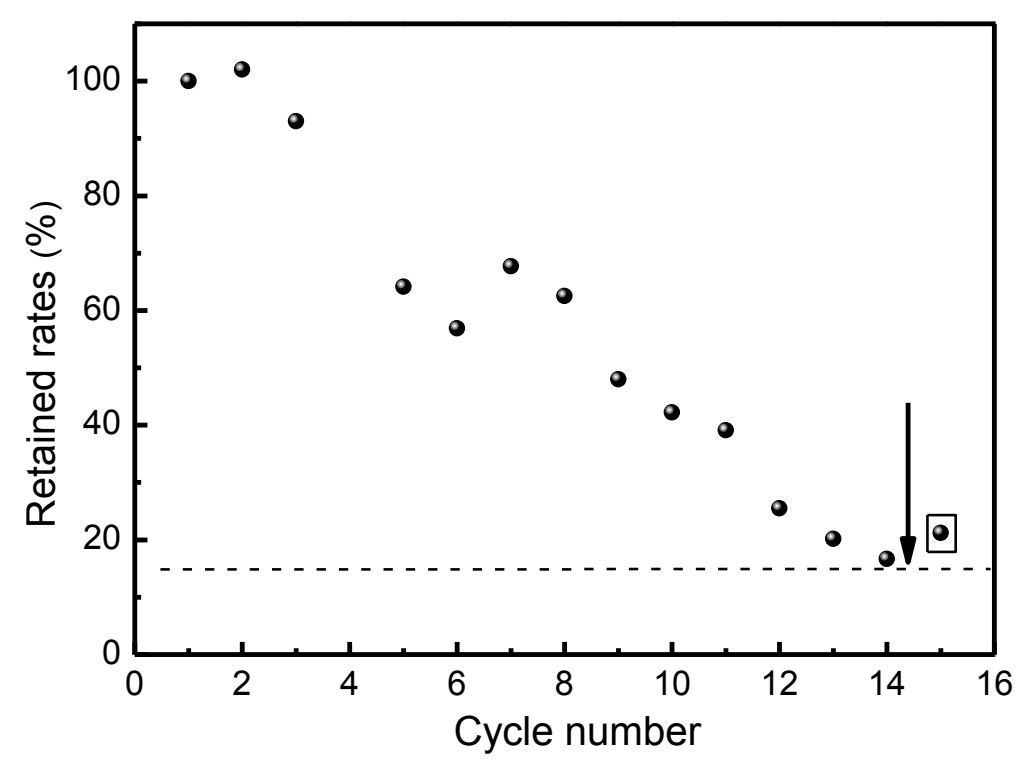


Figure 5

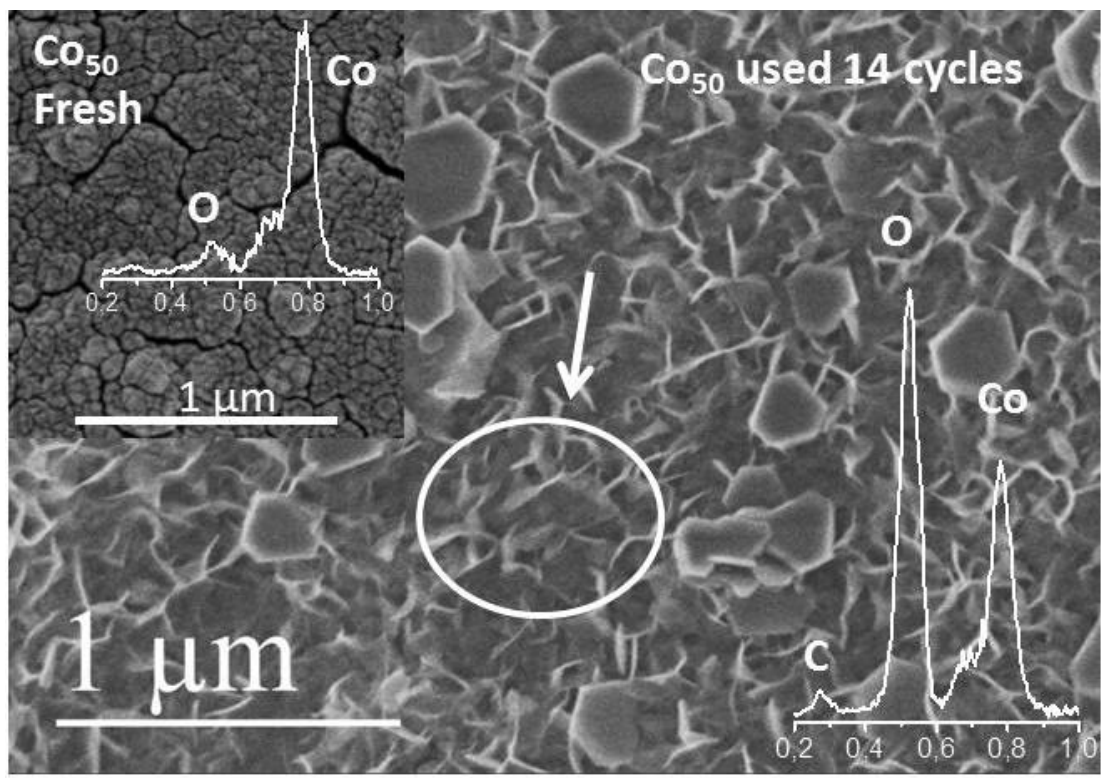


0
$\frac{1}{3}$
$\frac{0}{2}$
$\frac{1}{4}$
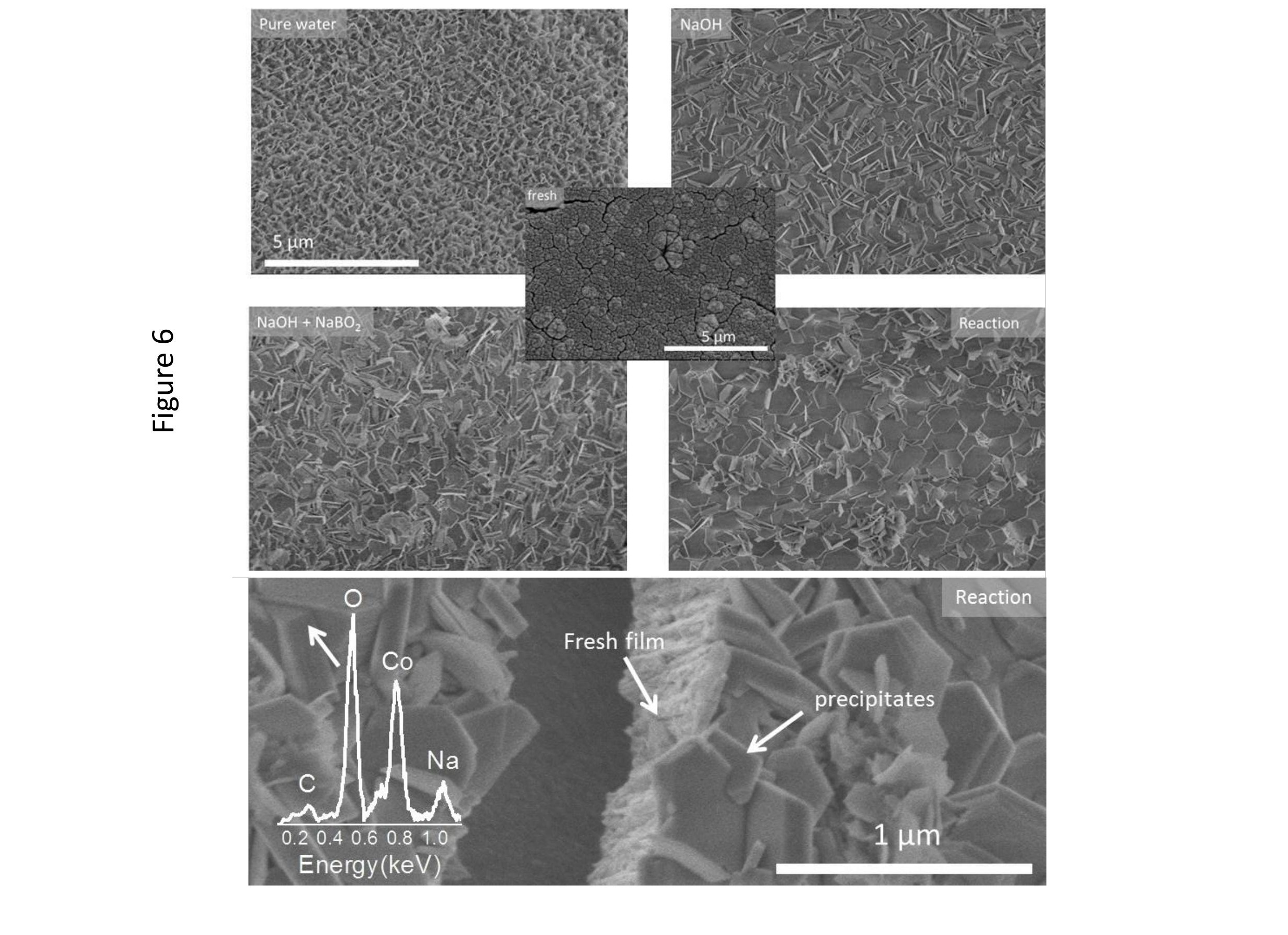
Figure 7

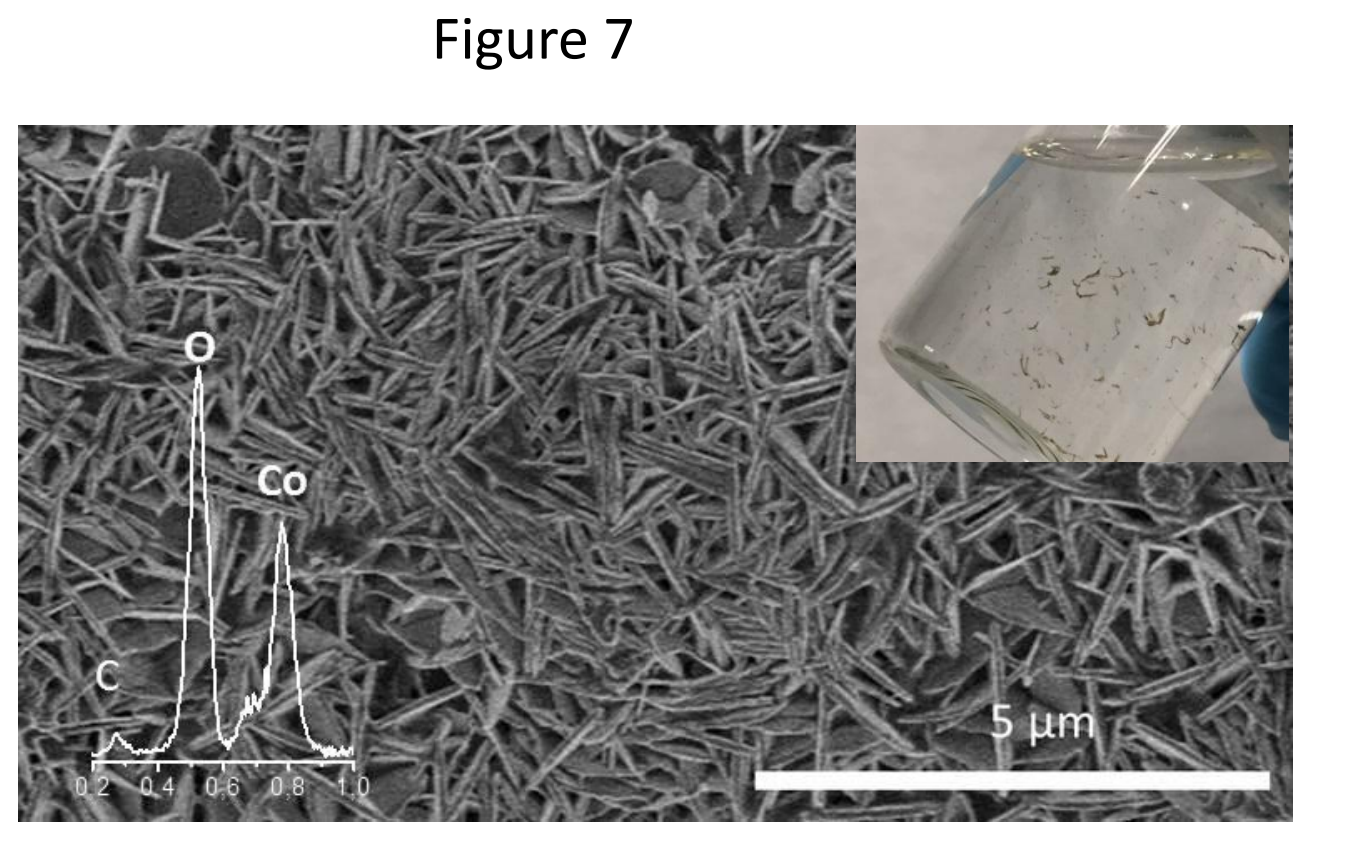

Fiqure

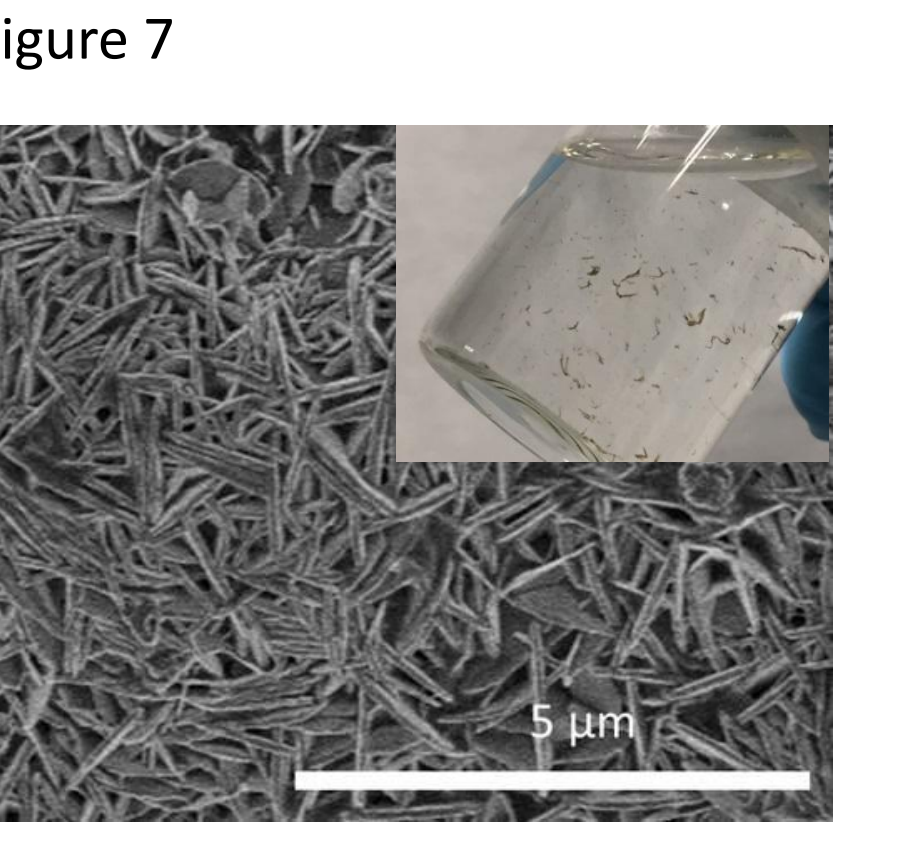

Figure

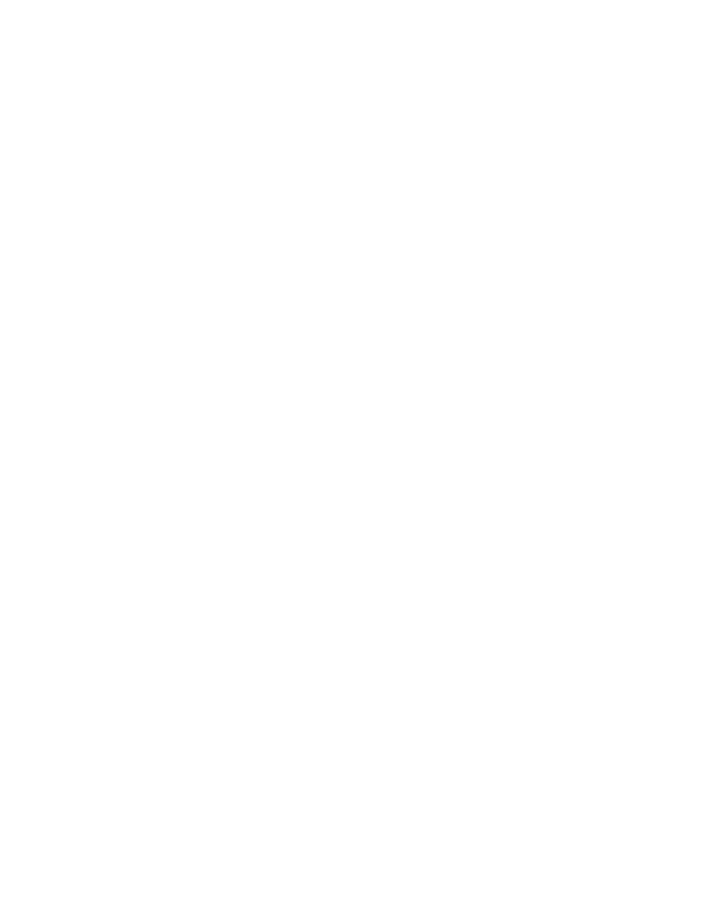

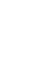

(
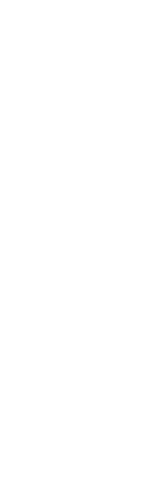


\section{Figure 8}
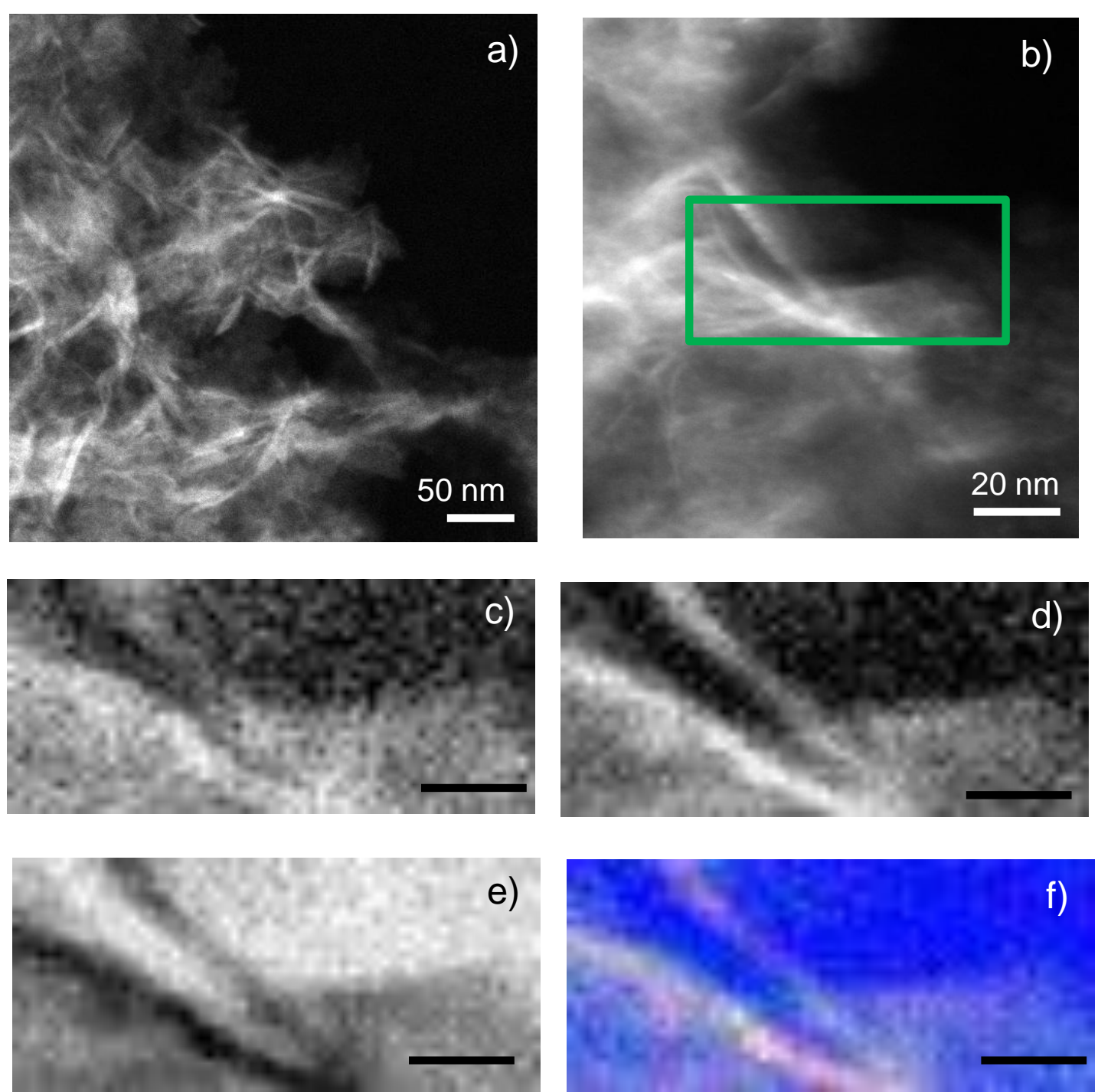

e)

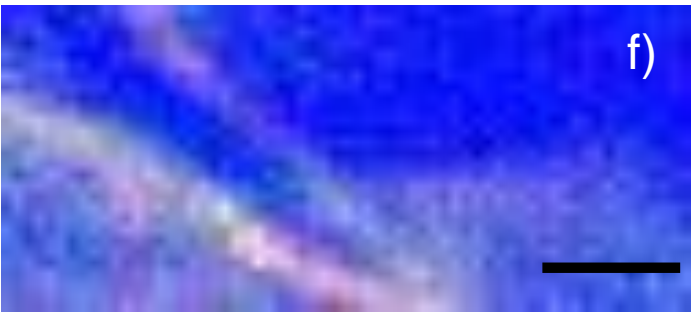




\section{Figure 9}
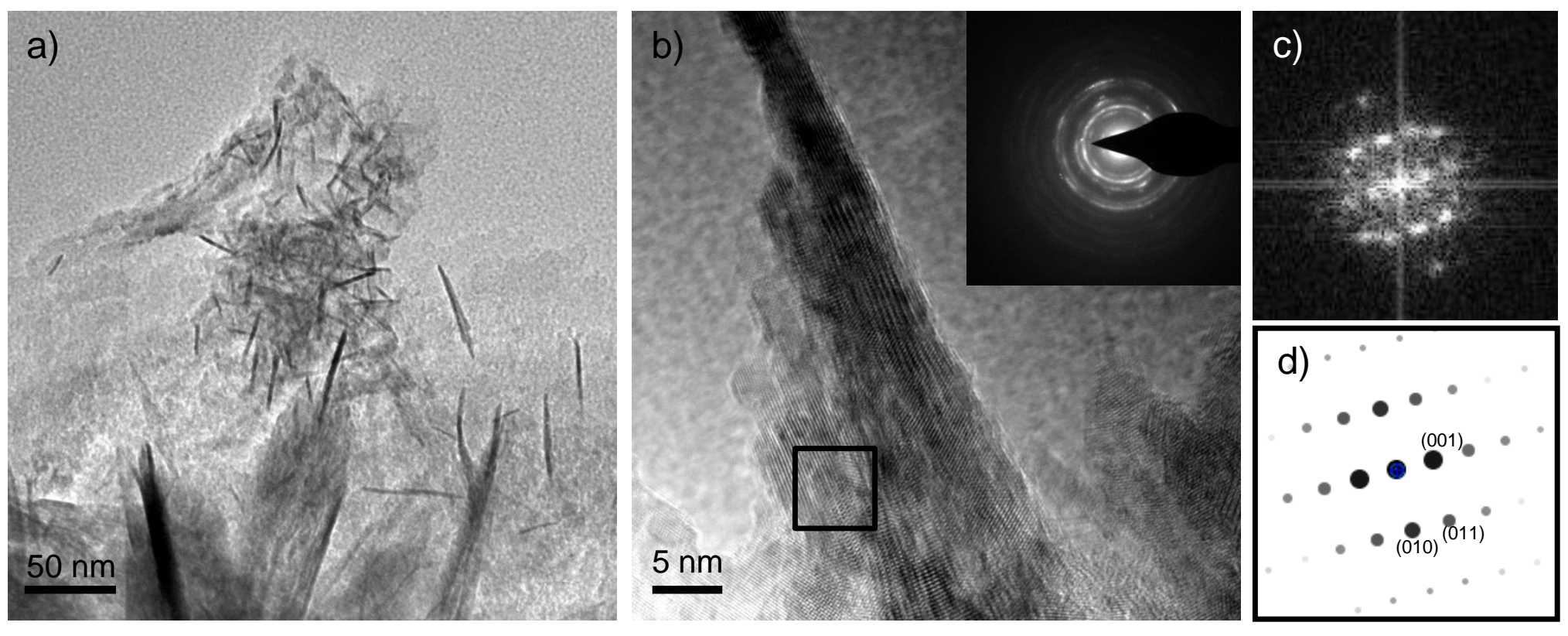
Figure 10

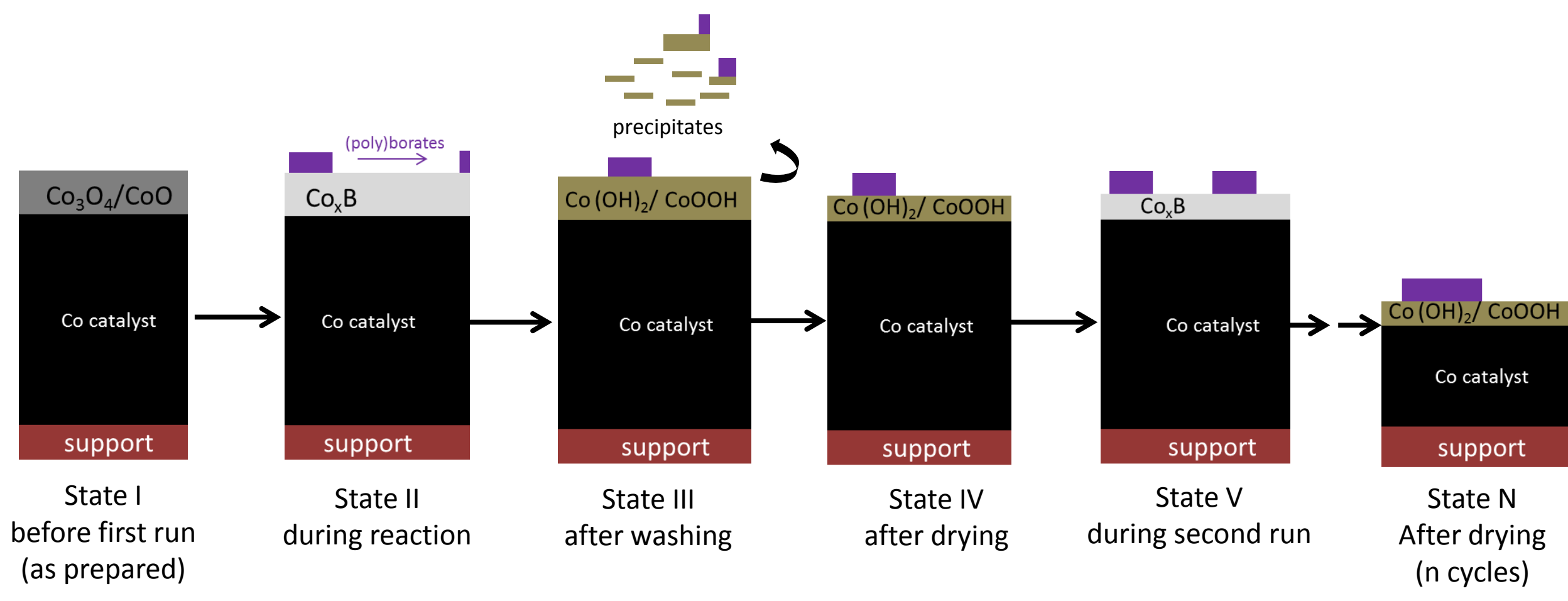

\section{State III after washing}

State IV after drying After drying (n cycles)




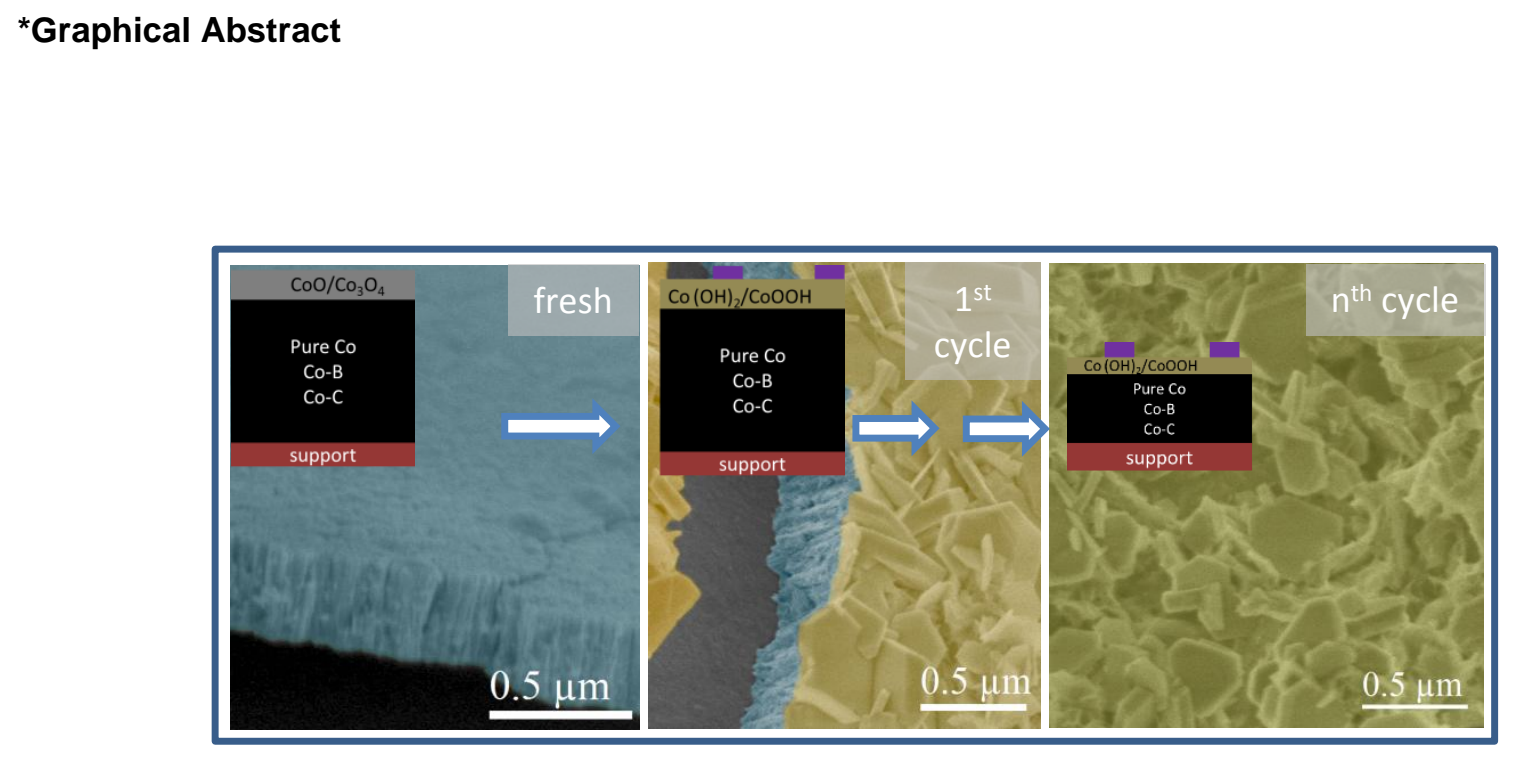


Deactivation was studied for a pure Co catalyst prepared by MS for SB hydrolysis

The formation of B-O based layer detected but not responsible of deactivation

Cobalt hydroxide and oxyhydroxide formed on catalyst and detached after reaction

Leaching of soluble cobalt negligible. Little physical thin film detachment

Same deactivation mechanism occurred for Co-B and Co-C samples 
Supplementary Material
Click here to download Supplementary Material: Revised Supporting information_d.pdf

Supplementary Material
Click here to download Supplementary Material: Revised Supporting information_d.pdf

\begin{abstract}
Click here to download Supplementary Material: Revised Supporting information d.pdr
\end{abstract}

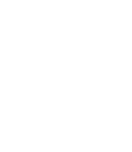

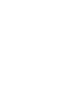

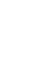

$\sqrt{10}$

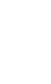
(1) (n) $\sqrt{20}$ (1) (1)

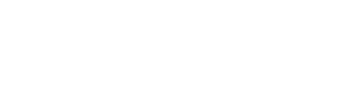

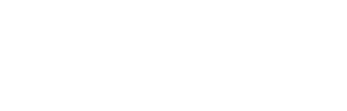
$x^{2}$

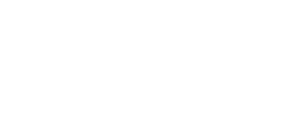

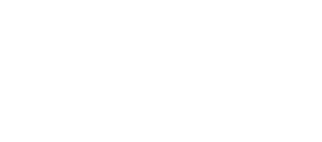
. (1) (1) (1) (1) (1) (1) (1) (1) (1) (1) (1) (1) (1)

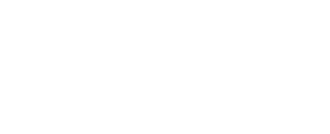

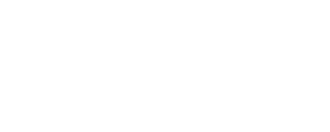

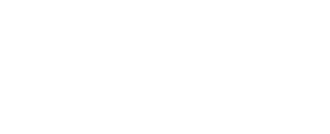
(2)

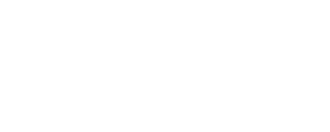
(1) (1) (1) 